\title{
Dysfunction of neurovascular/metabolic coupling in chronic focal epilepsy
}

Article

Accepted Version

Song, Y., Torres, R. A., Garcia, S., Frometa, Y., Bae, J., Deshmukh, A., Lin, W.-C., Zheng, Y. and Riera, J. J. (2016) Dysfunction of neurovascular/metabolic coupling in chronic focal epilepsy. IEEE Transactions on Biomedical Engineering, 63 (1). pp. 97-110. ISSN 0018-9294 doi: https://doi.org/10.1109/TBME.2015.2461496 Available at https://centaur.reading.ac.uk/67198/

It is advisable to refer to the publisher's version if you intend to cite from the work. See Guidance on citing.

Published version at: http://dx.doi.org/10.1109/TBME.2015.2461496

To link to this article DOI: http://dx.doi.org/10.1109/TBME.2015.2461496

Publisher: IEEE

All outputs in CentAUR are protected by Intellectual Property Rights law, including copyright law. Copyright and IPR is retained by the creators or other copyright holders. Terms and conditions for use of this material are defined in the End User Agreement.

\section{www.reading.ac.uk/centaur}

\section{CentAUR}


Central Archive at the University of Reading

Reading's research outputs online 


\title{
Dysfunction of Neuro-vascular/metabolic Coupling in Chronic Focal Epilepsy
}

\author{
Yinchen Song, Rafael A. Torres, Sarahy Garcia, Yisel Frometa, Jihye Bae, Abhay Deshmukh, Wei-Chiang Lin, \\ Ying Zheng and Jorge J. Riera*
}

\begin{abstract}
In this study, we aim to evaluate the mechanisms underlying the neuro-vascular/metabolic coupling in the epileptogenic cortices of rats with chronic focal epilepsy. To that end, we first analyzed intracranial recordings (electrophysiology, laser Doppler flowmetry and optical imaging) obtained from the seizure onset zones during ictal periods and then used these data to fit a metabolically-coupled balloon model. This biophysical model is an extension of the standard balloon model with modulatory effects of changes in tissue oxygenation, capillary dynamics and variable $\mathrm{O}_{2}$ extraction fraction. As previously reported using acute seizure models, we found that there is a significant higher contribution from high local field potential frequency bands to the cerebral blood flow (CBF) responses in the epileptogenic cortices during ictal neuronal activities. The hemodynamic responses associated with ictal activities were distance-dependent with regard to the seizure focus, though varied in profiles from those obtained from acute seizure models. Parameters linking the $\mathrm{CBF}$ and relative concentration of deoxy-hemoglobin to neuronal activity in the biophysical model were significantly different between epileptic and normal rats. In particular, we found that the coefficient associated with the strength of the functional hyperemic response was significantly larger in the epileptogenic cortices, although changes in hemoglobin concentration associated with ictal activity reflected the existence of a significantly higher baseline for oxygen metabolism in the epileptogenic cortices.
\end{abstract}

Index Terms-Chronic focal epilepsy, cerebral blood flow, oxygen consumption, metabolically-coupled balloon model, neuro-vascular/metabolic coupling, preclinical model.

\section{INTRODUCTION}

$\mathrm{M}$ ANY epileptic patients have refractory epilepsy, in which seizures are not controlled by medications. Neurosurgery has become increasingly feasible and successful, as seizure-freedom is achieved in $50 \%-60 \%$ of the cases. This surgical procedure relies upon accurate resection of the seizure onset zones. To help localize and delineate these zones during pre-surgical work-up, surgeons use a range of neuroimaging technologies, including EEG-fMRI; MRS with $1 \mathrm{H}$-NAA, 11C-choline; PET- with FDG (18F) \& FMZ, AMT(11C) ligands, ictal-perfusion SPECT with 99mTc (ECD \&

This work was supported by Florida International University start-up package to JJR. Asterisk indicates corresponding author.

Y. Song, R.A. Torres, S. Garcia, Y. Frometa, J. Bae, A. Deshmukh, W.C. Lin and J.J. Riera are with the Department of Biomedical Engineering at Florida International University, 10555 West Flagler Street, EC 2610, Miami, FL 33172, USA (correspondence e-mail: jrieradi@ fiu.edu).

Y. Zheng is with the School of Systems Engineering at University of Reading, Whiteknights, Reading, Berkshire, UK.
HMPAO), and video EEG/MEG with current source localization [1, 2]. Over the last decade, EEG-fMRI technique has been progressively introduced in hospitals [3-6]. This technique offers good sensitivity (88\%) and specificity (75\%) in localizing epileptogenic cortices in patients with focal refractory epilepsy [7]. By using EEG-fMRI technique, we can generate whole-brain maps of blood oxygenation level-dependent (BOLD) activations evoked by interictal epileptiform discharges (IEDs), which are useful to identify irritative zones. The pathogenic relationships between the irritative zones and the seizure-onset zones are poorly understood in focal epilepsy [8]. Nevertheless, IEDs are accepted as specific EEG markers of interest for pre-surgical evaluation of epileptic patients, because they are very frequent paroxysmal and asymptomatic events, are easily recorded and provide high specificity for localizing seizure-onset zones [9].

A phenomenon known as functional hyperemia constitutes the fundamental principle underlying the EEG-fMRI technique. The hyperemic response is an increase in cerebral blood perfusion in any particular brain region whenever the activity of neurons in this region suddenly augments from its baseline. A variety of physiological mechanisms have been proposed to account for the communication between neurons and their surrounding vasculature [10]. Increase in blood perfusion causes decrease in the relative concentration of deoxy-hemoglobin ([Deoxy-Hb]) due to an augment in cerebral blood volume (CBV) at the level of post-capillary venous. This produces an early and pronounced overshoot in the BOLD response. A delayed undershoot in the BOLD response is being associated with increases in the oxygen metabolism associated with the enhancement in neuronal activity. Canonical hemodynamic response functions (HRFs) used in most of the available software to perform BOLD signal analysis (SPM, BrainVoyager, and FSL) capture well typical over/under-shoot components in the BOLD signal. In fMRI analysis, HRFs are convolved with the neuronal activity to create area-dependent regressors of a general linear model.

Dysregulations in the mechanisms for neuro-vascular/metabolic coupling have been demonstrated to exist in epileptogenic brain areas [11-16], which might lead to abnormal HRFs. For example, pre-ictal vasoconstrictions in areas near the epileptogenic zones have been found in these previous studies. Also, it has been shown that the hyperemic response and the neurovascular unit are compromised during seizure episodes. As for healthy cortical regions, direct relationships between the gamma band of the local field potential (LFP) and the cerebral blood flow (CBF) during ictal 
periods have been demonstrated. All these previous studies have been performed using acute models of epilepsy, in which seizures are generated by local application of 4-aminopyridine (4-AP) or bicuculline. Unfortunately, such local applications of epileptic promoters are not able to induce long-term changes in the cortical microcircuits, microvasculature and metabolic networks that result from seizure establishment and perpetuation. Therefore, conclusions from such acute models should be carefully interpreted, and will require future validations, first in chronic preclinical models of epilepsy and later in epileptic patients. In addition, abnormal increases in the BOLD signal undershoots, in some cases causing an apparent negative BOLD response, have been reported in epileptogenic cortices [17-20]. This phenomenon has been associated with dysregulations in oxygen metabolisms within epileptogenic cortices. Therefore, the HRFs must have unique temporal profiles in chronic epileptogenic cortices. Some methods have been developed in the past to estimate the HRF voxel-wisely $[21,22]$. However, these methods lack of appropriate sensitivity to detect the small changes in BOLD signals associated with IEDs. In our opinion, parametric methods, such as the metabolically-coupled balloon model [23], properly adjusted to data recorded from chronic epileptogenic cortices will definitely help increase accuracy in the fMRI analysis. Failing to take account of the impaired neurovascular coupling and abnormal metabolic rates in the epileptic brain might affect seizure onset zone localization in epileptic patients who are candidates for ablative neurosurgery, precluding EEG-fMRI use.

Dysregulations in the neuro-vascular/metabolic coupling might be proportionately reflected in both IED and ictal events, but the latter provide much better signal-to-noise ratios (SNR) to quantify it. Unfortunately, IEDs are small events which cause hemodynamic and metabolic changes difficult to be detected with current invasive recording in animal models; i.e., laser Doppler flowmetry (LDF) and intrinsic optical signal imaging (IOSI). In this study, we evaluated several aspects of the neuro-vascular/metabolic coupling during ictal activity in chronic epileptic rats, with emphasis on: a) neuronal activity features underlying the functional hyperemic response, b) heterogeneities in the cortical micro-vascular organization with respect to the seizure initiation zones, and c) unbalance in the perfusion/metabolic rates resulting from sustained neuronal activation, which might be a factor for seizure establishment and perpetuation. To that end, we used a model of chronic pharmacoresistant focal epilepsy, which consists of first creating cortical malformations by exposing rats prenatally to methylazoxymethanol acetate (MAM) and later triggering recurrent seizures with pilocarpine. Substantial evidence indicates that this preclinical model mimics the relevant human pathophysiology [24]. Localizing the seizure onset zones constitutes the most challenging aspects of using a chronic preclinical model of epilepsy. In this paper, we localized cortical irritative zones by performing brain current source imaging from scalp EEG recording [25]. Then, we obtained invasive intracranial recordings (electrophysiological - LFP; optical - CBF, CBV and [Deoxy-Hb] through a craniotomy created on top of these irritative zones. We introduced a strategy to determine whether these irritative zones were focus for epileptogenic activity or not. Finally, we used a biophysical model to evaluate possible unbalances between the perfusion and metabolic rates during both brief and prolonged neuronal activation period.

\section{MATERIALS AND METHODS}

\section{A. The metabolically-coupled balloon model}

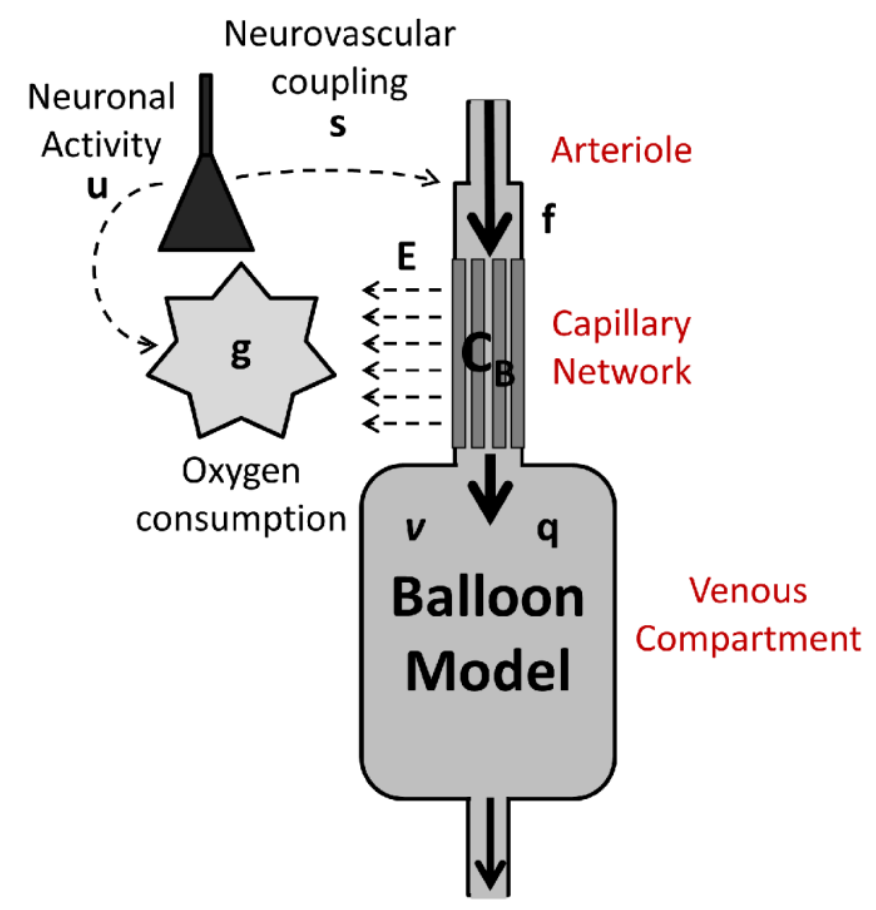

Fig. 1 Illustration of Metabolically-coupled Balloon Model

The metabolically-coupled balloon model (Fig. 1) [23] is an extension of the standard balloon model [26], which includes modulatory effects of changes in tissue oxygenation, capillary dynamics and variable $\mathrm{O}_{2}$ extraction fraction. Taking into consideration previous reports about possible dysfunctions in the hyperemic response and oxygen metabolism in epileptogenic cortices [11-16, 27], parameters $\varepsilon$ and $\kappa$ are of great interest in our study. $\varepsilon$ is an unknown parameter in the model relating neuronal activity to the CBF. Therefore, this parameter accounts for the actual strength of the hyperemic response. The larger the parameter $\varepsilon$ is, the greater the CBF. $\kappa$ is a scaling constant that links the neuronal activity to the changes in oxygen consumption. The larger the parameter $\kappa$ is, the higher the oxygen consumption. Therefore, we assumed that these two parameters might absorb important changes in the HRF temporal profiles in epileptic cortices. In addition, the final sign of the BOLD response will depend on the $\mathrm{O}_{2}$ consumption rate by neurons, as indicated by the ratio

$$
\vartheta=\varepsilon / \kappa,
$$

with a BOLD activation for $\vartheta>>1$ and a BOLD deactivation for $\vartheta<<1$. Therefore, fitting these two parameters ("Estimate Parameters", Table I) using intracranial recording might 
provide a physiological interpretation for the negative BOLD signal associated with IEDs. Based on previous studies, we don't expect significant changes in any other parameter of the metabolic-coupled balloon model ("Known Parameters", Table I). For those parameters, we used values reported by Zheng et al. obtained using IOSI from rats undergoing whisker stimulation [23]. According to our knowledge, this biophysical model has not been applied to explain the BOLD deactivation effect in epilepsy.

TABLE I

SUMMARY OF THE DYNAMICS EQUATIONS AND STATE VARIABLES FOR THIS MODEL.

\begin{tabular}{|c|c|c|c|}
\hline \multirow{2}{*}{ Equation } & \multirow{2}{*}{ Description } & \multicolumn{2}{|c|}{ Parameters } \\
\hline & & Known & Estimate \\
\hline$\dot{s}=\varepsilon u-s / \tau_{s}-(f-1) / \tau_{f}$ & Flow Inducing Signal & $\left(\tau_{s}, \tau_{f}\right)$ & $\varepsilon$ \\
\hline$\dot{f}=s$ & CBF & NA & NA \\
\hline$\mu \dot{g}=\left[\left(c_{B}-g c_{B}^{a}\right) / \beta-1\right]-\kappa u$ & Tissue $\mathrm{O}_{2}$ Level & $\left(c_{B}^{a}, \beta, \mu\right)$ & $\kappa$ \\
\hline$\varphi / f \dot{c}_{B}=-c_{B}-c_{B}^{a} E / \ln (1-E /(1-g))+c_{B}^{a} g$ & Capillary $\mathrm{O}_{2}$ Level & $\left(c_{B}^{a}, \varphi\right)$ & NA \\
\hline$\varphi / f \dot{E}=-E+(1-g)\left[1-(1-\xi)^{1 / f}\right]$ & $\mathrm{O}_{2}$ Extraction Fraction & $(\xi, \varphi)$ & NA \\
\hline$\tau_{0} \dot{v}=f-v^{1 / \alpha}$ & $\mathrm{CBV}$ & $\left(\tau_{0}, \alpha\right)$ & NA \\
\hline$\tau_{0} \dot{q}=f E / E_{0}-v^{(1-a) / \alpha} q$ & {$[$ Deoxy-Hb] } & $\left(\tau_{0}, \alpha, E_{0}\right)$ & NA \\
\hline \multicolumn{4}{|c|}{ Physiological values and definition of parameters $\mathrm{c}_{\mathrm{B}}{ }^{\mathrm{a}}$, and $\mathrm{g}_{0}$ are $\mathrm{c}_{\mathrm{B} 0}$ given in [23]. } \\
\hline \multicolumn{4}{|c|}{$\begin{array}{l}\varphi \text { determines whether the capillary dynamics and } \mathrm{O} 2 \text { extraction fraction follow a steady-state regime o } \\
\text { not. Parameters } \beta \text { and } \xi \text { are defined by the following formulas: }\end{array}$} \\
\hline \multicolumn{4}{|l|}{$\beta=c_{B_{0}}-g_{0} c_{B}^{a}$} \\
\hline \multicolumn{4}{|l|}{ and } \\
\hline$\xi=E_{0} /\left(1-g_{0}\right)$ & & & \\
\hline
\end{tabular}

In order to verify whether parameters $\varepsilon$ and $\kappa$ were statistically identifiable from CBF and [Deoxy-Hb] data, we performed a simulation study. Using actual inputs $\mathbf{u}$ for each trial obtained from epileptic rats, we integrated the metabolically-coupled balloon model using the local linearization method with different realizations for the observation and system noises [28]. We estimated the parameter $\varepsilon$ using only simulated $\mathrm{CBF}$ data. Parameter $\kappa$ was estimated using only the simulated [Deoxy-Hb] data. Parameter estimation was performed using the methodology proposed below (Materials and Methods, Section F-4). A total of 200 trials were performed for each parameter. Estimated values were then compared with actual values.

\section{B. Animal preparation}

All experimental procedures were approved by and carried out in full compliance with the Institutional Animal Care and Use Committee at Florida International University (Approval No. 13-065). All rats were received from Charles River Laboratories. The pharmacoresistant FCD preclinical model was established with male Wistar rats following the protocol described previously by Colciaghi et al. [24]. In brief, this protocol includes two major steps: first, the cortical malformations of this preclinical model were induced prenatally on Embryonic Day 15 with two doses of MAM injections $(15 \mathrm{mg} / \mathrm{kg}$ maternal body weight) 12 hours apart; second, 50 MAM-treated rats were intraperitoneally (i.p.) injected with pilocarpine (PILO, $300 \mathrm{mg} / \mathrm{kg}$ ) on Post-natal Day 28 to induce the status epilepticus (SE). All 50 MAM-PILO rats were monitored by four investigators (YS, RT, AD and JJR) independently to identify the SE according to the Racine scale [24]. Rats experiencing SE received phenobarbital $(20 \mathrm{mg} / \mathrm{kg})$
90 min after SE onset to reduce mortality, then were hydrated subcutaneously with Lactate Ringer's solution and fed by the investigators to improve the survival rate. Rats not experiencing SE were euthanized. Eventually, 13 MAM-PILO rats survived from this procedure and developed chronic seizures. From this point on, these 13 MAM-PILO rats will be referred to as epileptic rats. In addition, 4 normal male Wistar rats $(\sim 350 \mathrm{~g})$ were used as control in this study. All normal and epileptic rats were housed in standardized cages at a $12 \mathrm{~h}-12 \mathrm{~h}$ light-dark cycle with food and water ad libitum.

Rats were anesthetized with isoflurane (5\% for initial induction and then 1-2\%, 1 L/min $\mathrm{O}_{2}, 14 \mathrm{PSI}$ ) during all the manipulation and surgical procedures. During the following recording procedures (i.e., 32-channel scalp EEG and intracranial recordings), rats were anesthetized by a mixture of dexmedetomidine hydrochloride (Dexdomitor, $0.25 \mathrm{mg} / \mathrm{kg}$, i.p.) and low doses of isoflurane $\left(0.5-0.8 \%, 1 \mathrm{~L} / \mathrm{min}_{2}, 14 \mathrm{PSI}\right)$, which does not compress cortical hemodynamic responses [29-32]. The rat body temperature was maintained using a water-circulating heating pad (TPZ-0510EA, Texas Scientific Instruments, LLC) with a pump (TP700, Texas Scientific Instruments, LLC). The rat body temperature $\left(\sim 37^{\circ} \mathrm{C}\right)$, heart rate (200-300 beats per minute) and respiration rate $(<50$ breaths per minute) were monitored continuously by PowerLab 8/35 data acquisition device and LabChart software (ADInstruments) to assure the level of anesthesia remained stable throughout the entire experimental procedures.

\section{EEG Source Imaging: Identification of Irritative Zones}

1) Recording: The epileptic rat's scalp was shaved and cleaned with ethanol (90\%) for delipidation. A 32-channel EEG mini-cap specifically designed for rodents [33] was placed on top of the epileptic rat's scalp to record the EEG signals (PZ3, Tucker-Davis Technology). EEG signals were amplified, filtered $(0.5 \mathrm{~Hz}$ high-pass, and $250 \mathrm{~Hz}$ low-pass), and digitized (5 kHz sampling rate, $0.5 \mu \mathrm{V}$ resolution).

2) IED-related Primary Current Sources: From the averaged power of the 32-channel scalp EEG signals, IEDs, of which temporal patterns include spikes $(20-70 \mathrm{~ms}$ in duration, $15-50 \mathrm{~Hz}$ ) and sharp-waves (70-200 $\mathrm{ms}$ in duration, $5-15 \mathrm{~Hz}$ ), were identified by a combination of multiple band-pass/notch filters and an auto-thresholding method. The IED detection method is similar to those reported in human studies [34,35], as well as animal studies [25, 36]. Subsequently, spikes and sharp-waves were separated into multiple sub-types using a series of feature extraction and classification methods described previously [25, 36]. Each sub-type of IEDs was considered as a unique event. Event-related 32-channel EEG potentials were created by averaging the EEG data time-locked to the IED onsets within corresponding window size (80-ms for spikes and 200-ms for sharp-waves) (Fig. 2A). sLORETA distributed inverse solution was estimated from the event-related potentials for each IED sub-type (Fig. 2B) using Brainstorm [37]. For each epileptic rat, surgically accessible location for craniotomy (Fig. 2C) was determined based on the source locations at the initial phases of each IED sub-type. These source locations were associated with irritative zone. A 
T2-weighted MRI atlas for Wistar rats was used to calculate the electric lead fields [38]. This atlas is defined on the Paxinos and Watson's coordinate system [39], facilitating the exact demarcation of the region for the craniotomy.

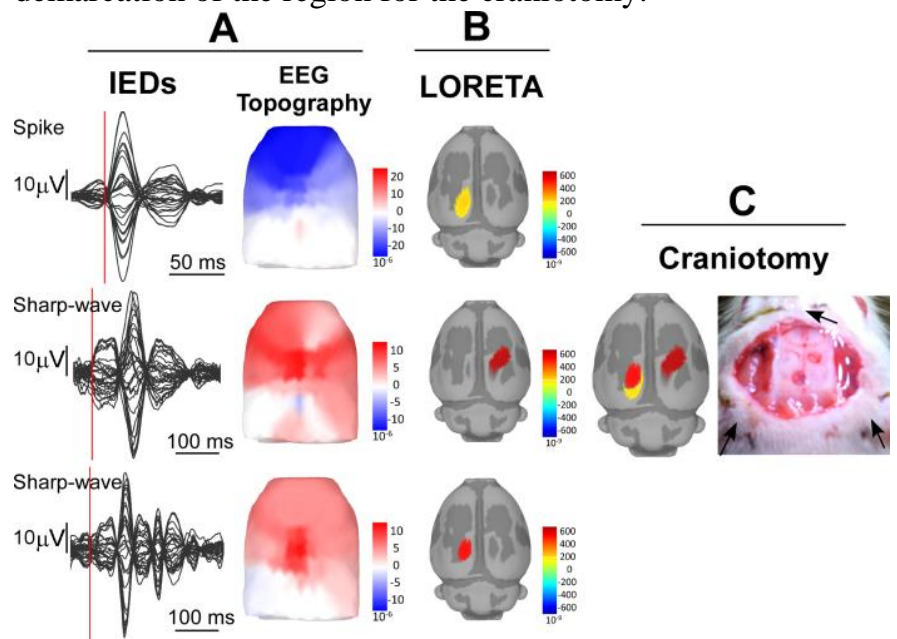

Fig. 2 Illustration of IED source imaging and craniotomy. (A) 32-channel IED waveforms (Spike (up) and Sharp-wave (middle and bottom)) and EEG topography for the initial phase latency (red vertical lines in (A)). (B) sLORETA inverse solution shows the source location of each type of IED. (C) Overlapping of all major sources identified in step ((B), left) and craniotomy on the rat (right). The black arrows ((C), right) are pointing out the locations where we inserted the carbon-filament electrodes subcutaneously.

\section{Forepaw stimulation on normal rats}

Forepaw stimulation was conducted only on normal rats. For the normal rats, the craniotomy location was on the right primary somatosensory cortex for forelimb (S1FL). Two needle electrodes were inserted into the left forepaw of the normal rat prior to the electrical stimulation and intracranial recordings. A block-design paradigm consisting of 10 blocks was employed. Each block was comprised of an $\mathrm{ON}$ period (i.e., simulation) and an OFF period (i.e., resting). For each rat, two different combinations of ON and OFF periods (ON-OFF) were utilized in the paradigm: 5s-30s (i.e., short) and 30s-50s (i.e., long). The current stimulation was delivered by the isolated pulse stimulator (Model 2100, 110V, $60 \mathrm{~Hz}, \mathrm{~A}-\mathrm{M}$ Systems) with $10-\mathrm{ms}$ pulse-width and 2.0-mA current amplitude at $3 \mathrm{~Hz}$.

\section{E. Intracranial Recording}

1) Craniotomy: Rats were anesthetized and fixed to a stereotaxic apparatus. Subsequent to the removal of the skin and connective tissues on top of the skull, a craniotomy of 2-3 $\mathrm{mm}$ in diameter was performed above the area of interest (AOI, i.e., irritative zones for epileptic rats, S1FL for normal rats) assisted by a digital microscope (VCR-800, HIROX). Two screws, used as reference and ground for the electrophysiological recordings, were attached to the skull distant to the craniotomy site. Dura mater covering the AOI was carefully removed. Non-conductive light-oil drops were applied to the craniotomy to protect it from drying during intracranial recordings.

2) Electrophysiological recordings: High-resolution intracranial electrical recordings were obtained using amplifiers at $25 \mathrm{kHz}$ (PZ2, Tucker and Davis Technologies,
Inc. "TDT") connected by an optical fiber to a signal processing unit (RZ2, TDT) and by a coaxial cable to a preamplifier located inside an acute headstage. Local field potential (LFP, 0.7-170 Hz) was extracted online using a logic/symbolic programming language (OpenEx software, TDT). The silicon-based probe used in this study (A16, 16-channel planar probe, NeuroNexus Technologies) consists of 1 linear shank with 16 recording sites (microelectrodes, $100 \mu \mathrm{m}$ intervals). The microelectrode impedance typically ranged within the interval 0.69-0.84 M $\Omega$. By implanting this probe semi-perpendicular to the AOI, we were able to simultaneously record extracellular potentials from different sites covering mainly the supra-granular $(0-400 \mu \mathrm{m})$ and granular $(400-700$ $\mu \mathrm{m})$ cortical laminas of the AOI. The insertion process was accurately monitored by a multi-micromanipulator control system (MPC-200/ROE 200/MPC-385/MPC-325 Sutter Instruments). To perform EEG recording (Animal Bio Amp, AD Instruments) on epileptic rats, carbon-filament electrodes (1 $\mathrm{mm}$ in diameter, $8 \mathrm{~mm}$ in length, World precision Instruments) were placed subcutaneously on top of the rat skull and secured with cyanoacrylate adhesive (Leica Microsystem) as shown in Fig. 2C. Scalp EEG data was needed for IED detection and classification in different sub-types for epileptic rats, which was achieved by using the same procedure as described in Section $C$ for the 32-channel scalp EEG recordings. EEG recordings from normal rats during forepaw stimulation were not required in this study.

3) Laser Doppler flowmetry recordings: We used a laser-Doppler flowmetry system (LDF; PeriFlux 4001Master, Perimed, Sweden) to record the CBF in the AOI using a needle-type probe (Probe 411, Perimed). The tip of the needle was placed on top of the cortex at a 30-degree angle to avoid contact with the electrophysiological probe. The LDF recording systems were connected to the PowerLab 8/35 (PL3508/P, AD Instrument) and data storage was performed with the LabChart Pro software.

4) Optical imaging: With a dual-wavelength IOSI system we developed in-house, we observed temporal variations in the diffuse reflectance signal of the AOI at two particular wavelengths $(\lambda)-500 \mathrm{~nm}$ and $650 \mathrm{~nm}$. The IOSI system constitutes a noninvasive imaging device capable of capturing 7.5 frame/sec for each wavelength. The optical imaging was performed independent of the LDF recordings, and vice versa.

\section{F. Intracranial Data Analysis}

1) Pre-processing: Electrophysiological recordings were synchronized with LDF and IOSI data using external trigger signal. LFP and EEG were down-sampled at $500 \mathrm{~Hz}$. Optical imaging data were co-registered spatially and temporally. Artifacts on imaging data were separated and removed using PCA.

2) Evaluation Criteria for Successful IED Localization: We employed two criteria to evaluate whether the IED source localization was accurate for each epileptic rat. First of all, IED-like events identified from LFP recordings should also be observable in subcutaneous EEG recordings. To detect the IEDs in LFP, we used the auto-thresholding method described 
above in Section $\mathrm{C}$ on the summed power through all 16 channels of the silicon-based microelectrode probe. Then we classified them into multiple clusters based on their temporal profiles using the same clustering method. For each LFP cluster, we checked the consistency of the corresponding EEG profiles for each event. If consistent, we considered the first criterion fulfilled. Secondly, IED source locations should be close to the silicon-based microelectrode probe, which would be reflected as dipolar-like features in volumetric current source density. To perform current source density (CSD) analysis using the LFP recordings for each IED, we used the CSDplotter toolbox (version 0.1.1). Therefore, we excluded any epileptic rat that did not meet any of these two criteria. Evidence has shown that the irritative zone could also be responsible for the seizure genesis $[9,40]$. Therefore, by localizing the irritative zone, it is possible that we could identify the seizure onset zone. From the resulting group of previous evaluation procedure, only those experiencing seizure-like activities within the AOI during the intracranial recordings were included in the final analysis.

3) Post-processing: To obtain the power of LFP at different frequency bands (i.e., delta $1-4 \mathrm{~Hz}$, theta $4-8 \mathrm{~Hz}$, alpha $8-12 \mathrm{~Hz}$, beta $12-25 \mathrm{~Hz}$, gamma-low $25-70 \mathrm{~Hz}$ and gamma-high $70-170$ $\mathrm{Hz}$ ), we performed a time-frequency analysis with a moving window of $1000 \mathrm{~ms}$ and an increment of $100 \mathrm{~ms}$ using the "spectrogram" function in Matlab. In order to remove baseline spectral components, we averaged the power for each frequency using the 10 seconds outside the seizure/stimulation temporal window, and subtracted it from the entire time-frequency power spectrum map [41]. The hemodynamic parameters, such as total hemoglobin concentration $[\mathrm{Hb}]$, oxy-hemoglobin concentration [Oxy-Hb] and [Deoxy-Hb], were extracted from the IOSI data at $500 \mathrm{~nm}$ and $650 \mathrm{~nm}$ using the modified Beer-Lambert law [42, 43].

Although it lacks a biophysical basis, an autoregressive model with an exogenous source (ARX)

$$
y_{t}=\phi_{0}+\sum_{i=1}^{p} \phi_{i} y_{t-i}+\sum_{i=1}^{r} \psi_{i} u_{t-i}+\varepsilon_{t}
$$

was applied on the intracranial data to understand the relationships between neuronal activity and hemodynamic responses roughly, where $\mathbf{y}_{\mathbf{t}}$ could be one of the time series of $\mathrm{CBF},[\mathrm{Hb}],[\mathrm{Oxy}-\mathrm{Hb}]$ and [Deoxy-Hb] (i.e., hemodynamics) that were extracted close to the microelectrode probe's location, $\mathbf{u}_{\mathbf{t}}$ could be one of the time series of the power of LFP at different frequency bands (i.e., neuronal activities) as exogenous source, $\boldsymbol{\varphi}$ and $\boldsymbol{\psi}$ are coefficients for $\mathbf{y}_{\mathbf{t}}$ and $\mathbf{u}_{\mathbf{t}}$ with an order of $\mathbf{p}$ and $\mathbf{r}$, respectively. $\boldsymbol{\varepsilon}$ t represent the instrumental error. ARX model has been employed frequently in neuroscience research to estimate the hemodynamic response evoked by neuronal activity [44-46]. It allows characterization of the relationship between neuronal activity (i.e., input) and hemodynamic response (i.e., output) in terms of the impulse response function (IRF), without any prior assumptions or knowledge of other physiological parameters. Here, ARX
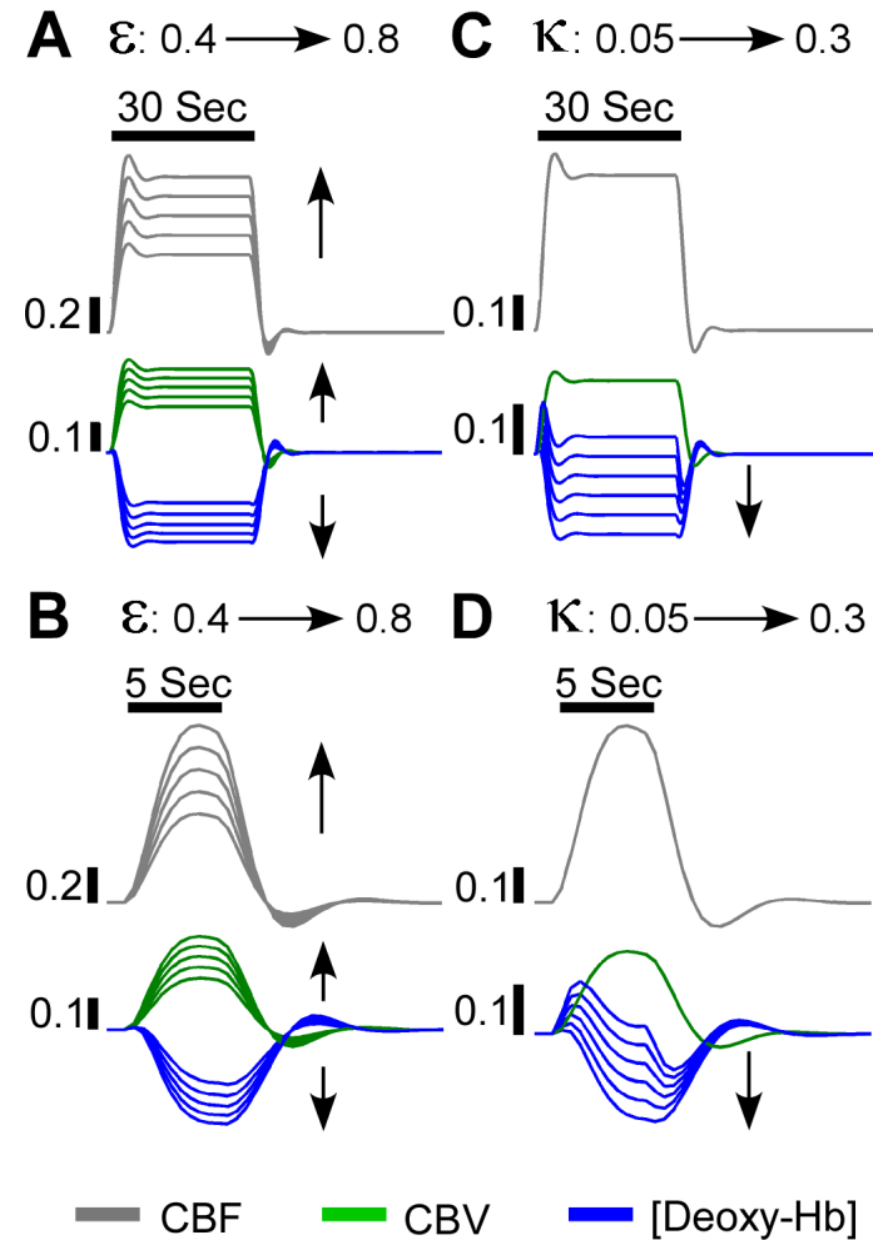

Fig. 3 Changes in the state variables of the metabolically-coupled balloon model when parameters $\varepsilon$ and $\kappa$ were varied. The parameter $\varepsilon$ was varied from 0.4 to 0.8 (step size: 0.1 ) for long (A) and short (B) periods of neuronal stimulation, and the parameter $\kappa$ was varied from 0.05 to 0.3 (step size: 0.05 ) for long (C) and short (D) period of neuronal stimulation.

orders $\mathrm{p}$ and $\mathrm{r}$ were estimated using the normalized root mean square error (NRMSE), which reflects the goodness-of-fitting. The ARX modeling offers a straightforward approach to evaluate the contribution from different frequency bands of the LFP to the respective hemodynamic response. The IRFs and optimal NRMSE of ARX model for each frequency band of LFP and the corresponding hemodynamic response were compared. We also investigated the spatial dependency of $[\mathrm{Hb}]$, [Oxy-Hb] and [Deoxy-Hb] IRFs with regard to the seizure initiation locations for the epileptic rats.

4) Parameter estimation with the metabolically-coupled balloon model: To estimate parameters $\varepsilon$ and $\kappa$ in the metabolically-coupled balloon model, we employed the local linearization filter and the innovation approach extended for heterogeneously sampled data, which is described in Riera et al. [47]. The instantaneous total power of LFP associated with both seizure episodes and forepaw stimulation was used as the input ut to the metabolically-coupled balloon model. Due to limitation of LDF and IOSI to observe vascular and metabolic changes in deep cortical layer, the LFP power was calculated using only the most superficial electrodes of the silicon-based microelectrode probe. We followed the same approach described in Section A to estimate $\varepsilon$ and $\kappa . \varepsilon$ was first estimated 
using the actual input $\mathbf{u}_{\mathbf{t}}$ for each trial and the corresponding CBF observations through the LDF technique. Subsequently, $\kappa$ was estimated using the actual input $\mathbf{u}_{\mathbf{t}}$ for each trial and the corresponding [Deoxy-Hb] observation through the IOSI technique. The estimated $\varepsilon$ and $\kappa$ from epileptic and normal rats were compared using a two-sample t-test in Minitab.

\section{RESULTS}

\section{A. Simulations based on metabolically-coupled balloon model}

Parameter $\varepsilon$ relates the neuronal activity to the normalized $\mathrm{CBF}$. While changing $\varepsilon$ alone, regardless of the duration of the neuronal stimulation, the $\mathrm{CBF}$ and $\mathrm{CBV}$ increase in amplitude linearly (Fig. 3A and 3B). [Deoxy-Hb] decrease as we increase the value of $\varepsilon$. Parameter $\kappa$ reflects changes in the $\mathrm{O}_{2}$ consumption rate associated with the neuronal activity. As illustrated in Fig. $3 \mathrm{C}$ and $3 \mathrm{D}$, when varying $\kappa$ alone, the $\mathrm{CBF}$ and CBV is not affected. However, [Deoxy-Hb] decrease as we increase $\kappa$.
A

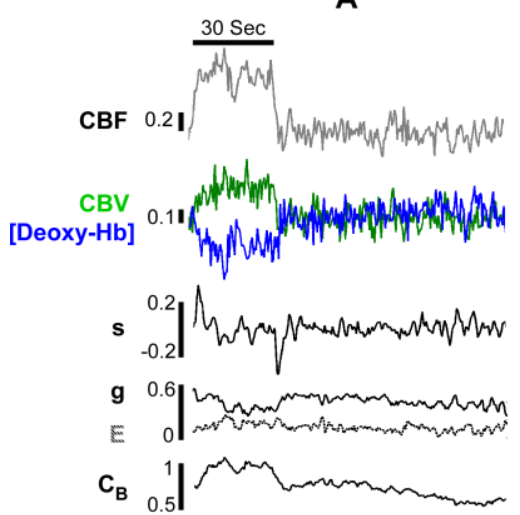

B

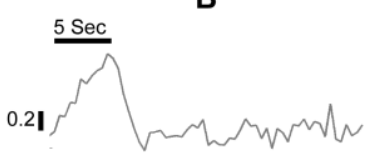

Fig. 4 Particular realizations of the state variables of the metabolically-coupled balloon model after introducing system and observation noises. Two particular durations of neuronal activation were used: (A) 30-second and (B) 5-second.
A

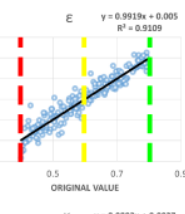

B

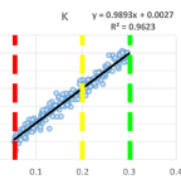

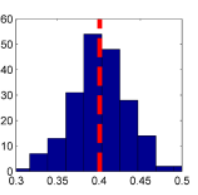

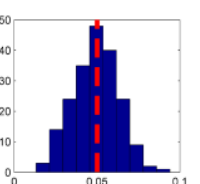

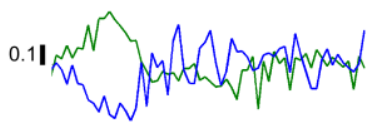
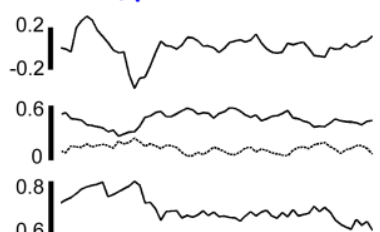

0.8
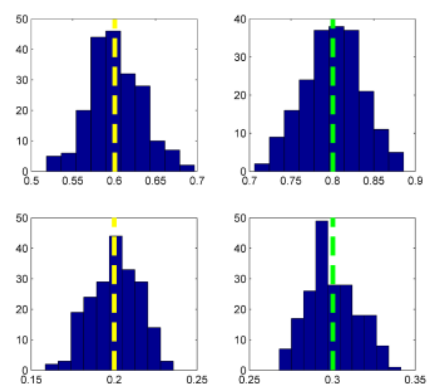

Fig. 5 Errors when estimating parameters $\varepsilon(\mathrm{A})$ and $\kappa(\mathrm{B})$. A significant linear relationship between the actual and estimated parameter are shown in the panels on the left. The distributions of the estimated parameter for three particular values are shown in the panels on the right. For each histogram, colored/dashed lines are used to indicate the actual value employed for each particular simulation. It can be noted that both parameters are estimated with high consistencies and low biases. The corresponding values in the linear regressions (left) are also indicated with colored/dashed lines. (Two Columns)

The biophysical model can be integrated using different realization of system and observation noises. Particular realizations for both short- and long- duration neuronal activity are shown in Fig. 4A and 4B. In this simulation, parameters $\varepsilon$ and $\kappa$ were varied from 0.4 to 0.8 and from 0.05 to 0.3 , respectively. For each value of these parameters, we created
200 trials of simulated data. The CBF and [Deoxy-Hb] simulated data were used to estimate parameters $\varepsilon$ and $\kappa$, respectively. These parameters were estimated with high consistency and low bias (Fig. 5), which indicates they are perfectly identifiable from the $\mathrm{CBF}$ and [Deoxy-Hb] data.

\section{B. Successful IED localization in epileptic rats}

Nine out of thirteen rats satisfied our evaluation criteria for successful IED localization using 32-channel EEG data. For each detected IEDs from the LFP in these 9 rats (Fig. 6A), we were able to consistently observe its reflections from the subcutaneous EEG (Fig. 6B), although as expected these two signals have different temporal profiles. In addition, the 16-channel LFP recordings from these 9 rats (Fig. 6C) had different temporal profiles with different polarities across electrodes, which indicates that the source of this particular type of IEDs was very close to the microelectrode probe as demonstrated in the CSD analysis (Fig. 6D). Among these nine rats, four of them happened to have focal ictal activities at the irritative-zone location (more than 5 seizures/rat within 30 minutes, see Supplementary Table I). Henceforth, we will focus on these four epileptic rats, and compare the results with those obtained using normal rats.

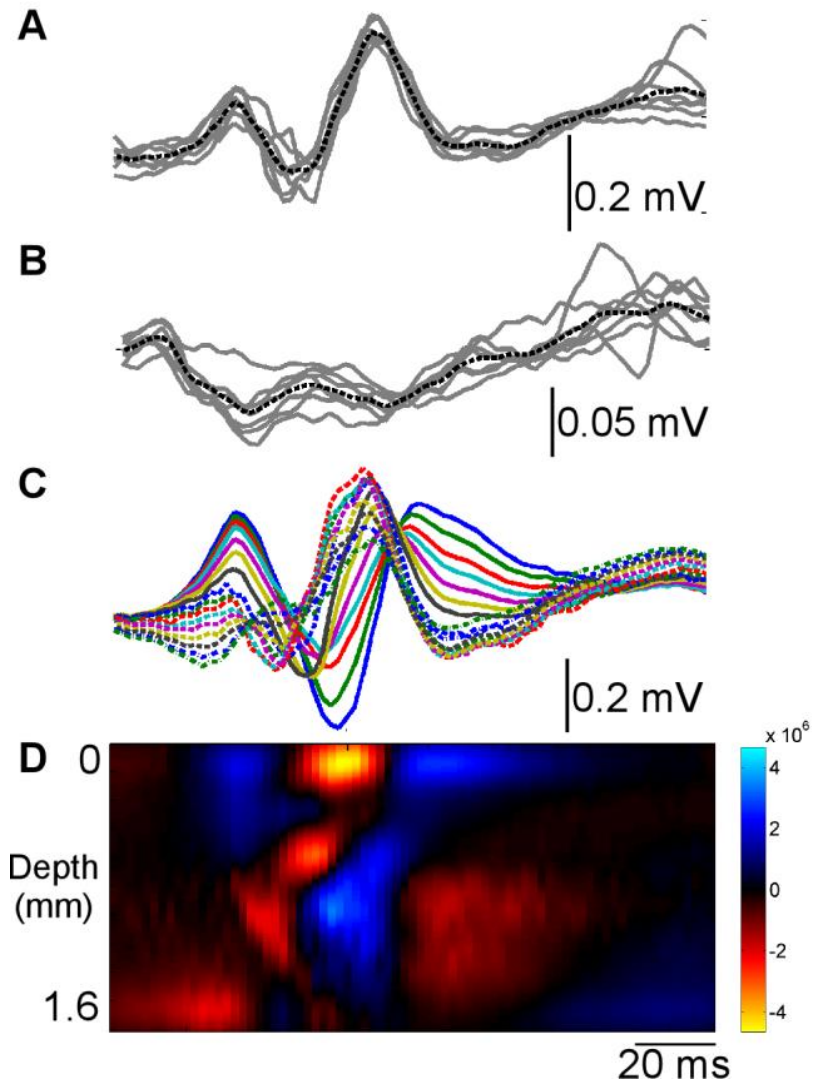

Fig. 6 Evaluation criteria for successful IED localization. (A) Each gray line is the averaged LFP through 16 channels for the same type of IEDs $(n=7)$, and the black dash line is the overall averaged LFP for this particular type of IEDs. (B) Each gray line is the subcutaneous EEG locked to the IED events detected in the LFP as (A) shown here. The black-dashed line is the averaged subcutaneous EEG for this type of IED. (C) 16-channel LFP signals averaged through 7 events are shown in different color and styles. (D) The current source density map (CSD) shows that the brain electrical source was very close to the silicon-based microelectrode probe, and part of it was localized in the superficial layers. 


\section{Functional Hyperemia during Seizures}

We observed significant $\mathrm{CBF}$ increases $(\mathrm{P}<0.05)$ on top of the irritative zones triggered by ictal LFP activities as shown in Fig. 7A. The CBF response did not show simultaneous increase when the seizure initiated, but it started to increase with a certain delay and then kept increasing until the end of seizure (Fig. 7A). It took a relatively longer time for CBF to return to the baseline. The power spectrum of LFP at different frequency bands varied during and after the seizure episode, some of which showed depression in power when seizure initiated and right after the end of the seizure (Fig. 7A). However, the contributions to the CBF response from each LFP frequency band were not equal. Beta and gamma bands had significantly higher contributions or relevance to the CBF response (Fig. 7B), since they could yield a much better fitting when used to estimate the CBF response with the ARX model. The IRFs for the $\mathrm{CBF}$ at each frequency band (Fig. 7C) were also obtained, respectively. IRFs at beta and gamma bands shared similar temporal profiles with relatively smaller standard deviations comparing to the IRFs for other LFP frequency bands. As mentioned in the Materials and Methods, the time-frequency power for each LFP frequency band was obtained using only the most superficial electrodes in the silicon-based microelectrode probe.

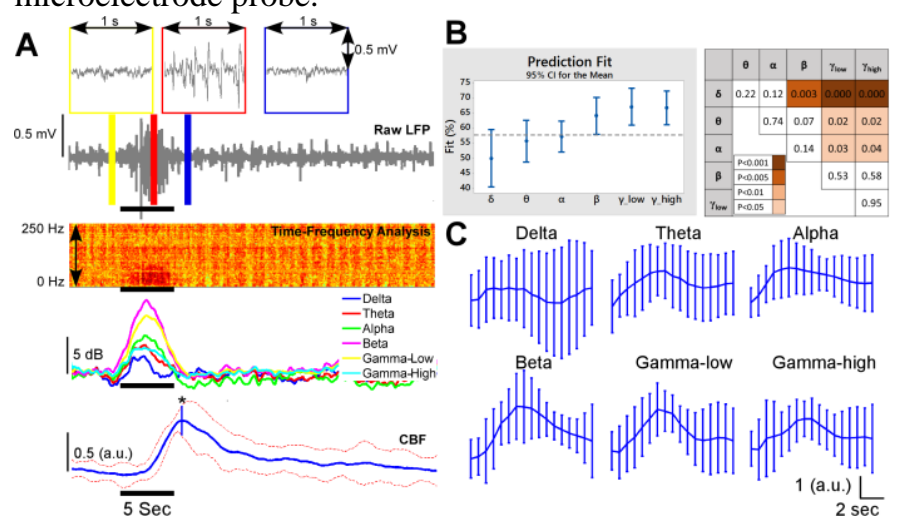

Fig. 7 Coupling between $\mathrm{CBF}$ and neuronal activity at different frequency bands. (A) A segment of raw LFP showing a seizure (black bar) as recorded from one superficial microelectrode in the silicon-based microelectrode probe is illustrated on the top panels. Enlarged versions (1-s) of the raw LFP for the pre-ictal, ictal and post-ictal windows are also presented in window with different colors (yellow, red and blue). A 2D color map shows the time-frequency composition of the LFPs for this particular trial (middle panel). Power of LFP at different frequency bands and averaged CBF response time-locked to ictal activity are shown in the bottom panels (averaged based on 9 ictal events from one of the epileptic rats). The decrease of CBF before the seizure onset, as previously reported [12], is not visible. The increase of CBF after the seizure onset is significant $(\mathrm{p}<0.05)$. (B) On the left, ARX model prediction fit at different frequency bands (based on 11 10-min recording segments from all 4 epileptic rats). The goodness of fitting at beta and gamma LFP frequency bands are much higher than those for other LFP frequency bands. On the right, Fisher individual tests for differences of mean prediction fits at different LFP frequency bands were conducted pair-wisely. The values shown in the blocks are the p-value. The blocks were colored with different darkness levels to represent the significance levels. (C) Impulse response function (IRF) at each LFP frequency band was estimated from the ARX model. (Two Columns)

\section{Spatiotemporal Hemodynamic Variations during Seizures}

$\mathrm{CBV}$ and [Deoxy-Hb] are two important hemodynamic characteristics that were monitored (in 2D images) using the IOSI system. From our IOSI recordings, we extracted time series for the CBV, [Oxy-Hb] and [Deoxy-Hb]. Subsequent to seizure initiation (around $260 \mathrm{~s}$ in Fig. 8A), CBV and [Oxy-Hb] started to increase while [Deoxy-Hb] is decreasing (Fig. 8C) within the area close to the microelectrode array. We also noted a depression in the power of LFP after the seizure episode around 360 second. Reflections of seizures within the optical field of view usually resembled an increase of CBV and a decrease of [Deoxy-Hb] with an epicentric origin followed by a peripheral propagation (Fig. 8D). Out-of-phase oscillations of $[\mathrm{Hb}]$ and $[$ Deoxy-Hb] reflected a common mechanism, i.e. decreases in the local [Deoxy-Hb] caused by relative enlargement of vessels volume after perfusion.

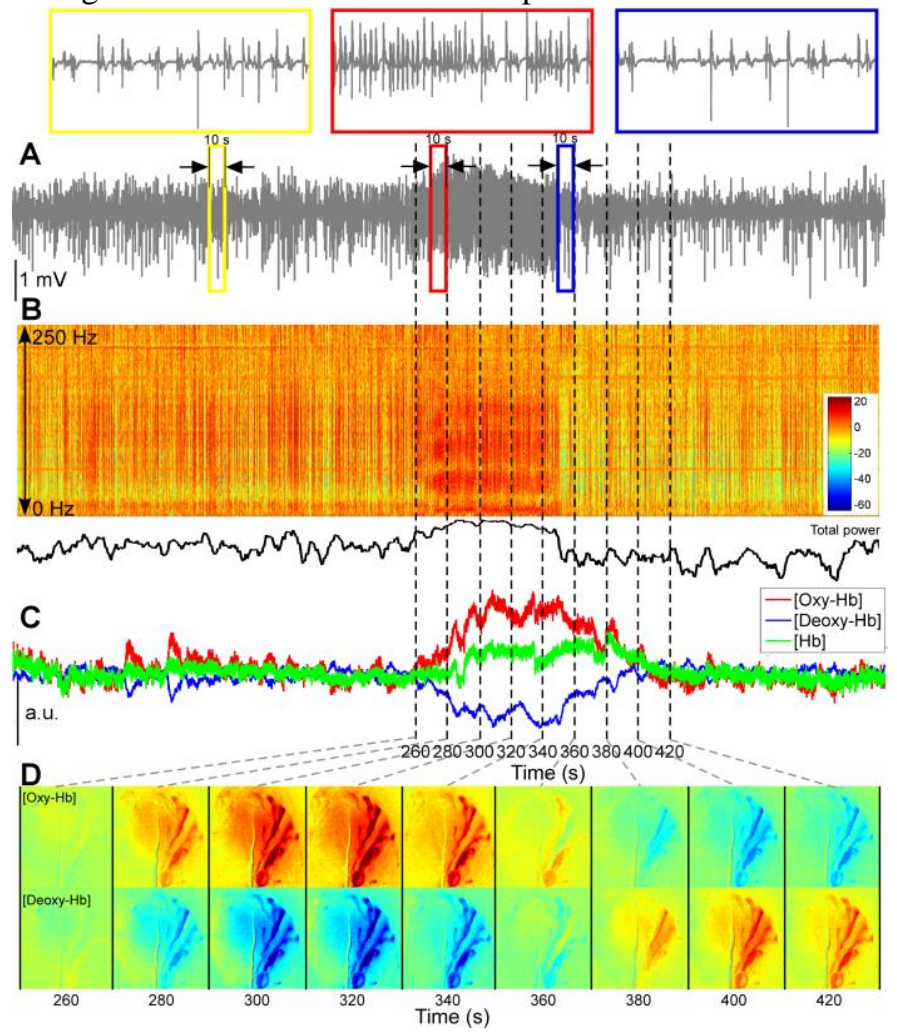

Fig. 8 Coupling between $[\mathrm{Hb}]$-related magnitudes and neuronal activity. The seizure initiated around 260 second. (A) The raw LFP recording with three 10-s windows showing activities before, during, and after the seizure episode. (B) The time-frequency analysis (top) and the total power spectrum $(0-250 \mathrm{~Hz}$, bottom) of a 10-min LFP recording. (C) The corresponding changes in [Hb], $[\mathrm{Oxy}-\mathrm{Hb}]$ and $[\mathrm{Deoxy}-\mathrm{Hb}]$ within the 10-min recording were extracted from the area close to insertion location of the silicon-based microelectrode probe. Significant variations were noticed after seizure initiation. (D) Spatial hemodynamic variations during the seizure. Image at each column corresponds to a particular time event marked with black dashed lines in (A) (B) and (C). Images at upper and lower panels show the variations in [Oxy-Hb] and [Deoxy-Hb], respectively. (Two Columns)

Besides the seizure focus, we also studied the CBV, $[\mathrm{Oxy}-\mathrm{Hb}]$, and [Dexoy-Hb] responses spatially as the seizure propagated from the focus. To that end, we used brain area without big vessels and selected a series of pixels at different locations along the propagation path from the seizure focus (Fig. 9A). These locations were separated into three geometric areas in terms of the distance to the seizure focus, named proximal, middle and distal areas. $\mathrm{CBV},[\mathrm{Oxy}-\mathrm{Hb}]$, and [Deoxy-Hb] time series from each selected pixel were calculated within a window of $5 \times 5$ pixels and averaged 
regarding the three geometric areas. The IRFs of $\mathrm{CBV}$, $[\mathrm{Oxy}-\mathrm{Hb}]$ and $[\mathrm{Deoxy}-\mathrm{Hb}]$ from all these selected pixels (Fig. 9B) and from those belonging to the proximal, middle and distal areas (Fig. 9C), respectively, were estimated as well. The amplitude of the IRFs reduced significantly as the seizure propagated from proximal to distal area. The [Deoxy-Hb] at the distal area slightly increased when seizure started, and then had a rapid decrease. Similar spatial-temporal patterns in IRFs of $\mathrm{CBV},[\mathrm{Oxy}-\mathrm{Hb}]$ and $[\mathrm{Deoxy}-\mathrm{Hb}]$ related to ictal activities were observed from all four rats.

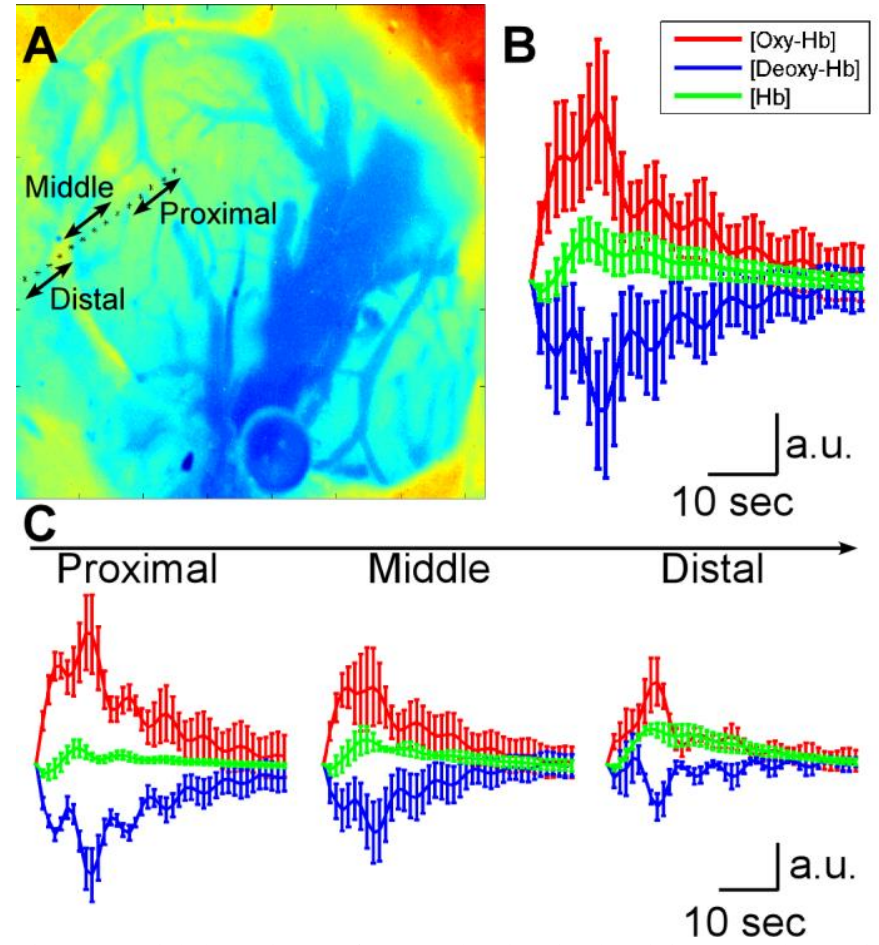

Fig. 9 Spatial dependency of IRFs related to ictal activity. (A) The spatial locations (dots) of the ( $5 \times 5$ pixels) areas at the proximal, middle and distal regions. (B) The IRFs of $[\mathrm{Hb}]$, [Oxy-Hb] and [Deoxy-Hb] obtained by pooling data from all regions in (A). (C) The IRFs of [Hb], [Oxy-Hb], and [Deoxy-Hb] obtained using only the corresponding areas for the proximal, middle and distal regions.

\section{E. Blood Perfusion v.s. Metabolic Rates}

Fig. 10 shows some examples of measurements obtained from both the epileptic rats with chronic seizures and normal rats that underwent electrical forepaw stimulation. Fig. 10A, $10 \mathrm{~B}, 10 \mathrm{E}$ and $10 \mathrm{~F}$ showed examples of simultaneous electrophysiological and LDF recording of ictal activity (A and B) and forepaw stimulation (E and F). Fig. 10C, 10D, 10G and $10 \mathrm{H}$ showed examples of simultaneous electrophysiological and IOSI recordings of ictal activity (on the seizure-initiation area, $\mathrm{C}$ and $\mathrm{D})$ and forepaw stimulation $(\mathrm{G}$ and $\mathrm{H})$. Note that a slight increase in [Deoxy-Hb] was visible at the beginning of each seizure episode; however, a decrease at the end of the stimulus episode, as predicted by the biophysical model for small $\kappa$ values (Fig. $3 \mathrm{C}$ and $3 \mathrm{D}$ ), was not clear.

We first used the LFP power (summed from 0 to $250 \mathrm{~Hz}$ ) and $\mathrm{CBF}$ for each trial of ictal activity $(\mathrm{n}=13$ - long latency, and $\mathrm{n}=17$ - short latency) and each trial of forepaw stimulation $(n=30-$ long latency, and $n=30-$ short latency) to estimate the parameter $\varepsilon$, as described in the Materials and Methods (Section
F-4). The variances of the estimated $\varepsilon$ from the epileptic cortex were much higher than those obtained from forepaw stimulation (Fig. 11, top-panel), reflecting larger inter-rat variability in the strengths of the functional hyperemic response in epileptic rats. There was no significant difference between values of $\varepsilon$ for long and short seizure duration (data not shown). Parameter $\varepsilon$ was significantly larger in epileptic cortices compared to normal cortices $(\mathrm{p}<0.001)$.

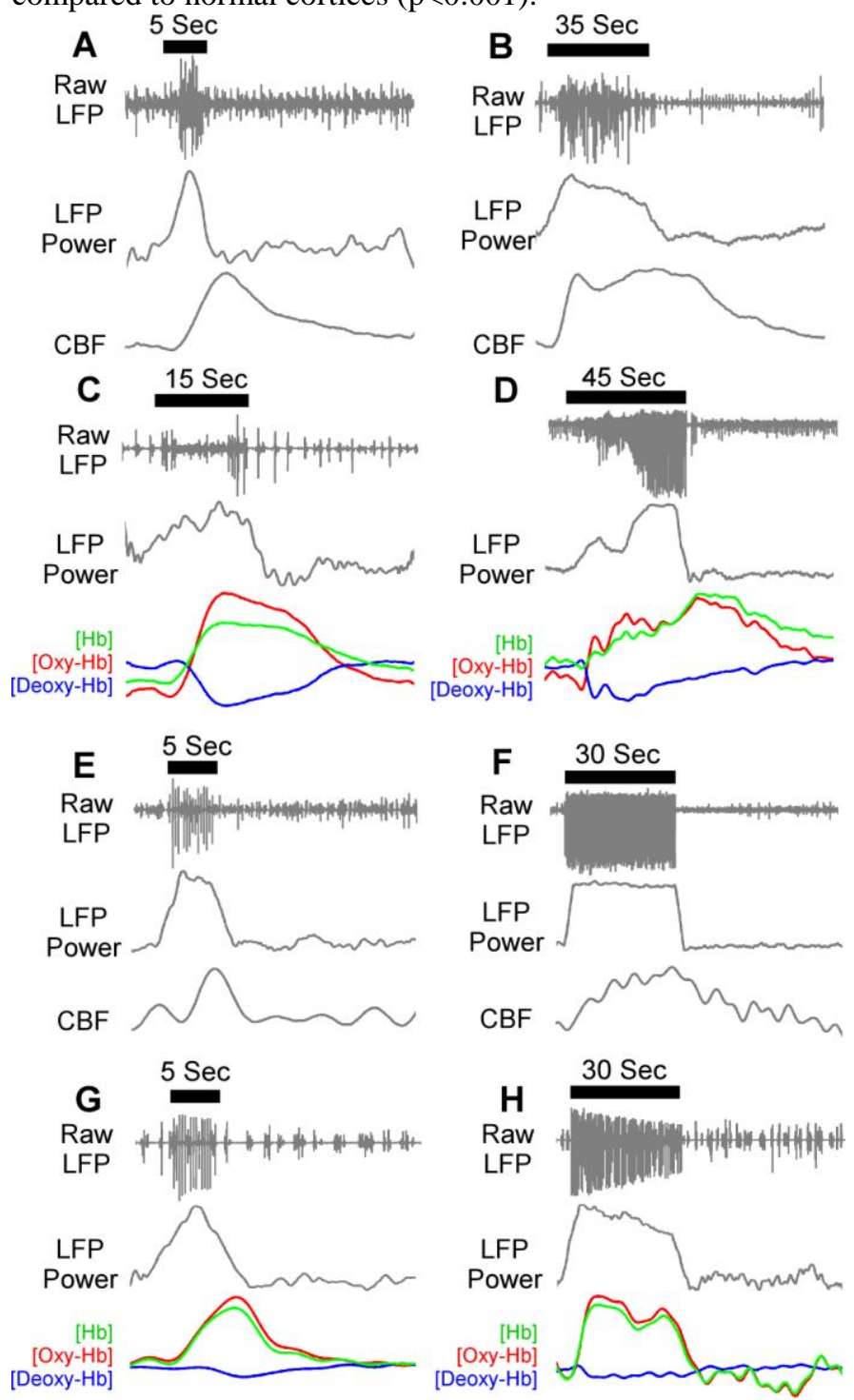

Fig. 10 Examples of neuronal activities and the respective hemodynamic responses for epileptic rats with different seizure durations and for normal rats undergoing electrical forepaw stimulation. For all cases, the raw LFP and its power $(0-250 \mathrm{~Hz})$ are shown on the top and middle of each panel, respectively. Either the $\mathrm{CBF}$ or the $[\mathrm{Hb}]$-related magnitude are shown on the bottom of each panel. (A-D): Ictal activity with both short and long duration from different epileptic rats. (E-H): Contralateral forepaw stimulation on normal rats with both short and long stimulation paradigms.

We used individual trials of LFP power (summed from 0 to 250 $\mathrm{Hz}$, CBV and [Deoxy-Hb] recording associated with both ictal activity (epileptic rats) and forepaw stimulation (normal rats) to estimate the $\kappa(n=9$ for ictal activity, and $n=60$ for forepaw stimulation). In this step, we used the mean value of the estimated parameter $\varepsilon$ for each individual rat. We also found larger variability in the values of parameter $\kappa$ for epileptic rats, 
compared to normal rats. The parameter $\kappa$ was significantly smaller in the epileptic rats $(\mathrm{p}<0.05)$, which in our opinion reflect higher baseline $\mathrm{O}_{2}$ metabolism epileptogenic areas (see Discussion).

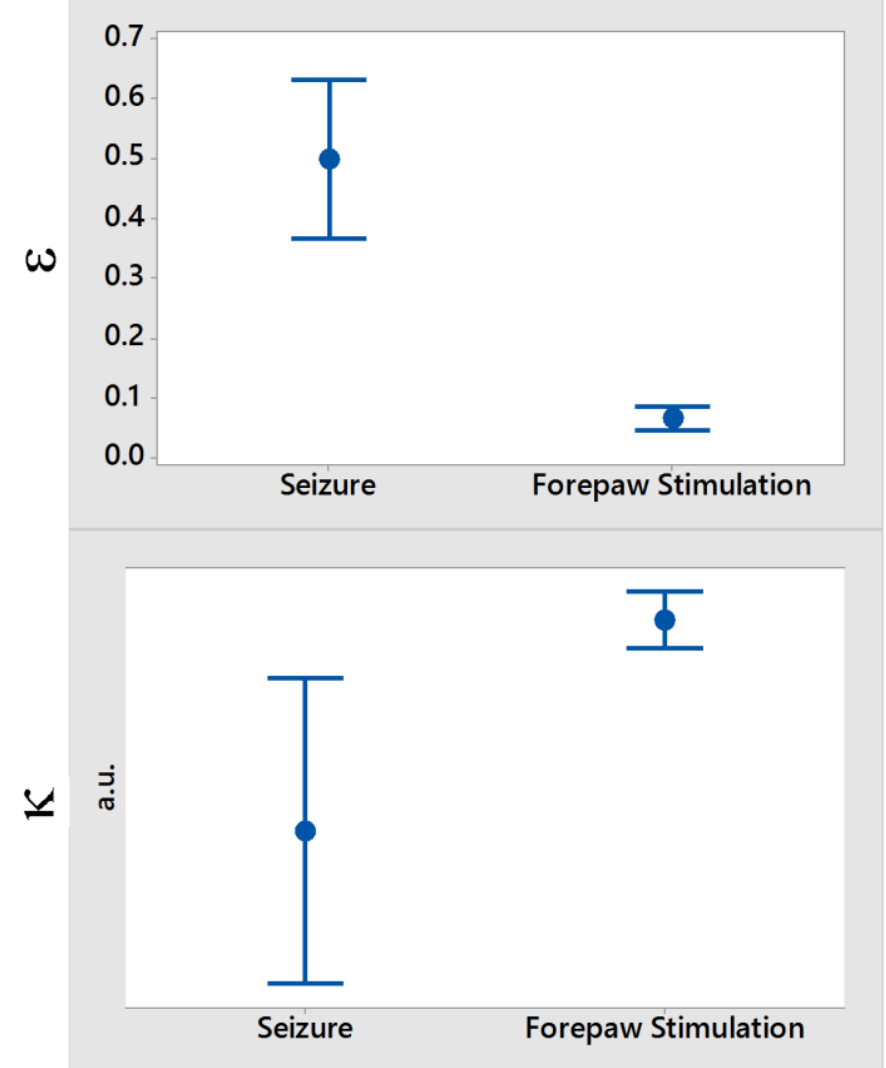

Fig. 11 Estimation of $\varepsilon$ and $\kappa$ based on intracranial recordings both from epileptic rats during seizure episodes and normal rats undergoing forepaw stimulation. Significant differences for both parameters $\varepsilon(\mathrm{p}<0.001)$ and $\kappa$ $(p<0.05)$ were found between epileptic rats and normal rats.

\section{DISCUSSION}

\section{A. Acute vs Chronic Model: Are the results comparable?}

In this study, we utilized a preclinical model of chronic epilepsy (MAM-PILO rat model) to investigate the dysfunction in neuro-vascular/metabolic coupling, instead of any acute model of seizures. Rats treated with MAM-PILO have grey matter heterotopias and malformations consisting of altered cortical lamination, and the presence of cytomegalic neurons with neurofilament over-expression, increased parvalbumin-positive puncta and misoriented dendrites. Similar hypertrophic/dysmorphic pyramidal neurons exist in acquired human FCD. GABAergic terminals enveloping the dysplastic neurons and NMDA receptor complex alterations observed in these neocortical abnormalities bear similarities with those observed in humans. Together, these features support the instrumental value of this preclinical model to analyze the pathogenesis of IED-evoked BOLD signal alterations in human FCD [48].

How do we reconcile our data based on chronic seizure model with those obtained from acute ones? We compared our results with those obtained from acute model in the following three aspects: a) the coupling between neuronal activity and hyperemic response; b) spatiotemporal hemodynamic variations with respect to the seizure focus; and c) the unbalance in the perfusion/metabolism rates resulting from the perpetuation of seizures.

First, Harris et al., has shown a powerful correlation between gamma-band $(25-90 \mathrm{~Hz})$ and CBV response in the acute model with 4-AP injections [13]. We demonstrated that, in addition to the lower part of gamma-band, the beta-band $(15-25 \mathrm{~Hz})$ and higher gamma-band $(70-170 \mathrm{~Hz})$ have much stronger contribution to the CBF responses than other frequency bands. Our findings might indicate that the electrophysiological basis of the acute model might be different from the one of chronic models.

Second, it has been demonstrated that a center-surround phenomena exist in the acute model of seizure, of which the observations include: 1) no decrease of CBF in the focus while a biphasic transient decrease of $\mathrm{CBF}$ existed in the surround [49]; 2) a transient dip in tissue $\mathrm{pO}_{2}$ followed by an increase in the focus while a sustained decrease of $\mathrm{pO}_{2}$ occurred in the surround [49]; 3) increase of metabolic rate of oxygen consumption in the focus while a sustained decrease in the surround (sometimes with a transient increase) [12]. These observations were supported by the active blood shunting theory through vasoconstriction in the surround to deliver oxygenated blood to the hyper-metabolic focus. However, an alternative hypothesis is also possible to explain it, which involves a decrease in neuronal activity in the surround area.

As in our studies, we observed significant spatiotemporal dynamics associated with ictal activity in terms of variations in $\mathrm{CBF}, \mathrm{CBV}$ and [Deoxy-Hb] as well: 1) within the seizure focus, there were no pre-ictal decreases in the CBF, and it did not start increasing immediately after the seizure initiated;2) We did not see any decrease at the beginning of CBV responses in the distal area caused by a pre-ictal vasoconstriction as reported in the acute models; 3) with ARX modeling, we observed an increase in [Deoxy-Hb] from the distal area after the seizure initiated followed by a rapid decrease afterwards, but the proximal area exhibited a sustained decreased in [Deoxy-Hb], which is contrary to the observation from acute models. Therefore, those hypotheses established on the basis of an acute model might not be sufficient to be associated with the chronic seizure model, and hence, the human epilepsy.

Third, comparing to the normal somatosensory stimulations, the transient decrease discovered in $\mathrm{pO}_{2}$ with the acute model is much larger (23\% vs. 3-4\%) [49, 50]. Our findings in the metabolically-coupled balloon model showed that the chronic seizure onset zones had significantly higher values of $\varepsilon$, which is a measure of the coupling between neuronal activity and $\mathrm{CBF}$, and lower values of $\kappa$, relating neuronal activity to [Deoxy-Hb], than the S1FL cortex with electrical forepaw stimulation. These two parameters could be used as promising biomarkers to identify epileptogenic areas in the epileptic patients with non-invasive EEG-fMRI analysis, instead of the current invasive techniques we used in this study.

In general, acute seizure model is a good approach to study the neuro-vascularlmetabolic coupling with highly controllable seizure induction. However, it cannot incorporate some 
developed pathophysiological conditions that epileptic patients have. Therefore, in order to have a close approximate to clinical epileptogenic conditions, we should use models with chronic epilepsy to investigate the coupling between neuronal activity and hemodynamiclmetabolic activities. In this case, there would be more confidence to translate the preclinical study into a clinical environment.

\section{B. Alterations in the HRF on epileptogenic cortices: Implication for the EEG-fMRI technique.}

Two parameters in the metabolically-coupled balloon model are of great interest in this study, which are $\varepsilon$ and $\kappa . \varepsilon$ relates the neuronal activity to the variations in CBF. On the other hand, $\kappa$ links the neuronal activity to the relative changes in [Deoxy-Hb]. However, we were able to obtain an absolute estimation of $\varepsilon$, while $\kappa$ was only relatively estimated at this point. This limitation was a consequence of using modified Beer-Lambert law to estimate the relative changes in [Deoxy-Hb]. Therefore, without a clear knowledge of the baseline level of [Deoxy-Hb] for each rat, it is impossible to derive an absolute estimation of $\kappa$ with current techniques.

We have proved that these two parameters are significantly different in the epileptogenic cortex, in comparison to the normal cortex (S1FL) undergoing forepaw stimulation. This implies that the neuro-vascular/metabolic coupling is altered in the epileptogenic brain. With respect to the exact alterations in the neuro-vascular/metabolic coupling, we noticed that $\varepsilon$ is significantly higher while $\kappa$ is significantly lower in the epileptogenic cortex. High $\varepsilon$ could be associated with the strengthened functional hyperemia during seizure activities, which has been reported in previous acute models of seizures [49]. In addition, it has been reported in preclinical studies that epileptogenic cortex has hypermetabolism [51, 52], as a result of which we speculate that the baseline metabolism in the epileptogenic cortex is relatively higher in comparison to the one in the normal brain. Therefore, even when there is increase in metabolism induced by the seizures, the metabolism in the epileptogenic cortex will not increase as much as it in the normal somatosensory cortex under stimulation, which will make the $\kappa$ relatively smaller. The following definition could be used to better understand this conjecture $\mathrm{\kappa u}_{\mathrm{t}}=\Delta \mathrm{M} / \mathrm{M}_{0}$, with $\Delta \mathrm{M}$ and $\mathrm{M}_{0}$ representing the relative change in $\mathrm{O}_{2}$ metabolism and its baseline, respectively. Therefore, a small $\kappa$ might be associated with either a small $\Delta \mathrm{M}$ or a large $\mathrm{M}_{0}$.

In our previous study [36], we conducted simultaneous EEG-fMRI recordings on six rats of this chronic epilepsy model and identified both positive and negative IED-evoked BOLD responses in the cortical areas using a canonical HRF [53] for estimation in SPM8. However, we were not able to obtain good significance with the correction for multiple comparisons. We used FMRISTAT [54] to retrieve the real HRFs from the areas with IED-evoked BOLD activation or deactivation and found that they actually shared different temporal profiles, which might explain why we could not obtain good significance with multiple comparisons. With the Granger causality analysis, we demonstrated that the brain areas with BOLD deactivation predominantly have directed influences on the area with activation, although they were not always within the closest vicinity. Hence, we separated these pairs of activation and deactivation with regard to their spatial profiles and found differences between the temporal profiles of those with negative HRFs, which might indicate different mechanisms underlying BOLD deactivation. IED-evoked BOLD deactivations have also been frequently reported in patients with focal epilepsy [55-57]. However, the mechanism underlying it and its potential effect on localizing irritative zones remain largely unknown.

To circumvent the potential problems with using normal canonical HRF to estimate the BOLD response in EEG-fMRI studies, multiple studies have demonstrated the possibilities of alternatives. One way people have been using to achieve this was to perform ten separate event-related analysis with HRFs consisting of a single gamma function peaking from -9 to 9 second around the IED events $[19,58]$. In such case, they were able to identify BOLD responses preceding the IEDs and found it frequently in patients with focal epilepsies [58]. In addition, they were more focal compared to the late response. Another method available is based on independent component analysis (ICA) without making any assumption of the profiles of HRFs [59] and the usage of EEG spatiotemporal patterns as event-related regressors. With parametric modeling, Storti et al. employed F-test and ICA criteria to select the most likely model from a pool of 5 parametric/generic HRFs [60]. However, Storti et al.'s HRF models were based on parametric mathematical models without links to actual pathophysiological mechanisms, which is the same for the other two methods; hence, they lack interpretation to understand the physiological basis of HRF dysregulations in focal epilepsy.

In this study, we demonstrated that we could use the metabolically-coupled balloon model to estimate the parameter $\varepsilon$ and $\kappa$ for different neuronal activities and found significant differences between the ictal activities in epileptic rats and somatosensory activation in normal rats. As we mentioned previously, these two parameters could be potentially useful to delineate the epileptic cortex from the normal one intraoperatively for epileptic patients undergoing surgical resection. With the estimation of parameter $\varepsilon$ and $\kappa$, the final sign of the BOLD response will depend on the $\mathrm{O}_{2}$ consumption rate by neurons, as indicated by the ratio in Eq. (1), with a BOLD activation for 1 and a BOLD deactivation for 1 . To validate this, we are working on a new study with simultaneous EEG-fMRI recordings to determine the irritative zones with IED-evoked BOLD activation and deactivation. Following that, we will perform the intracranial recordings within those areas in particular and estimate the parameter $\varepsilon$ and $\kappa$, respectively. Therefore, we will advance qualitatively in the use of EEG-fMRI technique to study epileptogenesis by using an actual biophysical model, as the one discussed in this paper. In future studies, we will use parameters estimated here to construct a pool of potentials HRFs for epileptogenic cortices.

\section{CONCLUSION}

In this study, we conducted intracranial recordings to 
understand the neuro-vascular/metabolic coupling during ictal activity in chronic epileptic rats with the help of a metabolically-coupled balloon model. We were able to detect significant hemodynamic responses (i.e., $\mathrm{CBF}, \mathrm{CBV}$, [Deoxy-Hb] and [Oxy-Hb]) associated with ictal activities of different durations. The spatiotemporal-dependent IRFs of hemodynamics were found to be different from those obtained in acute seizure models reported by other groups, which might indicate more complicated mechanisms underlying the phenomena observed in the chronic epilepsy model. $\varepsilon$ and $\kappa$, two important parameters to link neuronal activity to CBF and oxygen consumption, were estimated from both epileptic and normal rats. There are significant differences in the values of $\varepsilon$ and $\kappa$ between the epileptic and normal rats. Introducing methods to estimate these parameters, which reflect the underlying physiology, would enhance our understanding of the neuro-vascularlmetabolic coupling in epileptic patients and improve the localization accuracy on irritative zones and seizure onset zones through neuroimaging techniques.

\section{ACKNOWLEDGMENT}

Because our rats have chronic epilepsy, their health condition is too delicate. Therefore, the authors would like to thank FIU veterinary Haratiu V. Vinerean and his team for providing extra care in this particular case.

\section{REFERENCES}

[1]H. T. Chugani, Neuroimaging in epilepsy: Oxford University Press, 2010. [2]F. Pittau, F. Grouiller, L. Spinelli, M. Seeck, C. M. Michel, and S. Vulliemoz, "The role of functional neuroimaging in pre-surgical epilepsy evaluation," Frontiers in neurology, vol. 5, 2014.

[3]E. Formaggio, S. F. Storti, A. Bertoldo, P. Manganotti, A. Fiaschi, and G. M. Toffolo, "Integrating EEG and fMRI in epilepsy," Neuroimage, vol. 54, pp. 2719-2731, 2011.

[4]M. Hauf, K. Jann, K. Schindler, O. Scheidegger, K. Meyer, C. Rummel, et al., "Localizing seizure-onset zones in presurgical evaluation of drug-resistant epilepsy by electroencephalography/fMRI: effectiveness of alternative thresholding strategies," American Journal of Neuroradiology, vol. 33, pp. 1818-1824, 2012.

[5]N. Jette and S. Wiebe, "Update on the surgical treatment of epilepsy," Current opinion in neurology, vol. 26, pp. 201-207, 2013.

[6]L. A. van Graan, L. Lemieux, and U. J. Chaudhary, "Methods and utility of EEG-fMRI in epilepsy," Quantitative imaging in medicine and surgery, vol. 5, pp. 300-312, 2015.

[7]D. An, F. Fahoum, J. Hall, A. Olivier, J. Gotman, and F. Dubeau, "Electroencephalography/functional magnetic resonance imaging responses help predict surgical outcome in focal epilepsy," Epilepsia, vol. 54, pp. 2184-2194, 2013.

[8]M. de Curtis and G. Avanzini, "Interictal spikes in focal epileptogenesis," Progress in neurobiology, vol. 63, pp. 541-567, 2001.

[9]S. Tousseyn, P. Dupont, K. Goffin, S. Sunaert, and W. Van Paesschen, "Correspondence between large - scale ictal and interictal epileptic networks revealed by single photon emission computed tomography (SPECT) and electroencephalography (EEG)-functional magnetic resonance imaging (fMRI)," Epilepsia, vol. 56, pp. 382-392, 2015.

[10] J. J. Riera and A. Sumiyoshi, "Brain oscillations: ideal scenery to understand the neurovascular coupling," Current opinion in neurology, vol. 23, pp. 374-381, 2010.

[11] H. Ma, M. Zhao, and T. H. Schwartz, "Dynamic neurovascular coupling and uncoupling during ictal onset, propagation, and termination revealed by simultaneous in vivo optical imaging of neural activity and local blood volume," Cerebral Cortex, vol. 23, pp. 885-899, 2013.

[12] M. Zhao, J. Nguyen, H. Ma, N. Nishimura, C. B. Schaffer, and T. H. Schwartz, "Preictal and ictal neurovascular and metabolic coupling surrounding a seizure focus," The Journal of Neuroscience, vol. 31, pp. 13292-13300, 2011.

[13] S. Harris, H. Ma, M. Zhao, L. Boorman, Y. Zheng, A. Kennerley, et al., "Coupling between gamma-band power and cerebral blood volume during recurrent acute neocortical seizures," NeuroImage, vol. 97, pp. 62-70, 2014.

[14] S. Harris, L. Boorman, M. Bruyns - Haylett, A. Kennerley, H. Ma, M. Zhao, et al., "Contralateral dissociation between neural activity and cerebral blood volume during recurrent acute focal neocortical seizures," Epilepsia, vol. 55, pp. 1423-1430, 2014.

[15] N. Voges, S. Blanchard, F. Wendling, O. David, H. Benali, T. Papadopoulo, et al., "Modeling of the neurovascular coupling in epileptic discharges," Brain topography, vol. 25, pp. 136-156, 2012.

[16] I. Vanzetta, C. Flynn, A. I. Ivanov, C. Bernard, and C. G. Bénar, "Investigation of linear coupling between single-event blood flow responses and interictal discharges in a model of experimental epilepsy," Journal of neurophysiology, vol. 103, pp. 3139-3152, 2010.

[17] A. Salek-Haddadi, B. Diehl, K. Hamandi, M. Merschhemke, A. Liston, K. Friston, et al., "Hemodynamic correlates of epileptiform discharges: an EEG-fMRI study of 63 patients with focal epilepsy," Brain research, vol. 1088, pp. 148-166, 2006.

[18] E. Kobayashi, A. P. Bagshaw, C. Grova, F. Dubeau, and J. Gotman, "Negative BOLD responses to epileptic spikes," Human brain mapping, vol. 27, pp. 488-497, 2006.

[19] R. Rathakrishnan, F. Moeller, P. Levan, F. Dubeau, and J. Gotman, "BOLD signal changes preceding negative responses in EEG - fMRI in patients with focal epilepsy," Epilepsia, vol. 51, pp. 1837-1845, 2010.

[20] F. Pittau, F. Fahoum, R. Zelmann, F. Dubeau, and J. Gotman, "Negative BOLD response to interictal epileptic discharges in focal epilepsy," Brain topography, vol. 26, pp. 627-640, 2013.

[21] Y. Lu, A. P. Bagshaw, C. Grova, E. Kobayashi, F. Dubeau, and J. Gotman, "Using voxel-specific hemodynamic response function in EEG-fMRI data analysis," Neuroimage, vol. 32, pp. 238-247, 2006.

[22] J. Riera, J. Bosch, O. Yamashita, R. Kawashima, N. Sadato, T. Okada, et al., "fMRI activation maps based on the NN-ARx model," Neuroimage, vol. 23, pp. 680-697, 2004.

[23] Y. Zheng, J. Martindale, D. Johnston, M. Jones, J. Berwick, and J. Mayhew, "A model of the hemodynamic response and oxygen delivery to brain," Neuroimage, vol. 16, pp. 617-637, 2002.

[24] F. Colciaghi, A. Finardi, A. Frasca, S. Balosso, P. Nobili, G. Carriero, et al., "Status epilepticus-induced pathologic plasticity in a rat model of focal cortical dysplasia," Brain, vol. 134, pp. 2828-2843, 2011.

[25] J. Bae, A. Deshmukh, Y. Song, and J. Riera, "Brain Source Imaging in Preclinical Rat Models of Focal Epilepsy using High-Resolution EEG Recordings," JoVE, p. e52700, 2015/06/06/ 2015.

[26] K. J. Friston, A. Mechelli, R. Turner, and C. J. Price, "Nonlinear responses in fMRI: the Balloon model, Volterra kernels, and other hemodynamics," Neurolmage, vol. 12, pp. 466-477, 2000.

[27] S. Harris, M. Bruyns-Haylett, A. Kennerley, L. Boorman, P. G. Overton, H. Ma, et al., "The effects of focal epileptic activity on regional sensory-evoked neurovascular coupling and postictal modulation of bilateral sensory processing," Journal of Cerebral Blood Flow \& Metabolism, vol. 33, pp. 1595-1604, 2013.

[28] R. Biscay, J. Jimenez, J. Riera, and P. Valdes, "Local linearization method for the numerical solution of stochastic differential equations," Annals of the Institute of Statistical Mathematics, vol. 48, pp. 631-644, 1996.

[29] B. Bernal, S. Grossman, R. Gonzalez, and N. Altman, "FMRI under sedation: what is the best choice in children?," Journal of clinical medicine research, vol. 4, p. 363, 2012.

[30] E. Jonckers, J. Van Audekerke, G. De Visscher, A. Van der Linden, and M. Verhoye, "Functional connectivity fMRI of the rodent brain: comparison of functional connectivity networks in rat and mouse," PloS one, vol. 6, p. e18876, 2011.

[31] C. P. Pawela, B. B. Biswal, A. G. Hudetz, M. L. Schulte, R. Li, S. R. Jones, et al., "A protocol for use of medetomidine anesthesia in rats for extended studies using task-induced BOLD contrast and resting-state functional connectivity," Neuroimage, vol. 46, pp. 1137-1147, 2009.

[32] F. Zhao, T. Zhao, L. Zhou, Q. Wu, and X. Hu, "BOLD study of stimulation-induced neural activity and resting-state connectivity in medetomidine-sedated rat," Neuroimage, vol. 39, pp. 248-260, 2008.

[33] J. Riera, A. Sumiyoshi, R. Kawashima, "Electroencephalographic electrode unit for small animals and measurement system using same," US Patent, 2010.

[34] C. Pedreira, A. Vaudano, R. Thornton, U. Chaudhary, S. Vulliemoz, H. Laufs, et al., "Classification of EEG abnormalities in partial epilepsy with 
simultaneous EEG-fMRI recordings," NeuroImage, vol. 99, pp. 461-476, 2014.

[35] R. Quiroga, Z. Nadasdy, and Y. Ben-Shaul, "Unsupervised spike detection and sorting with wavelets and superparamagnetic clustering," Neural computation, vol. 16, pp. 1661-1687, 2004.

[36] Y. Song, B. G. Sanganahalli, F. Hyder, W.-C. Lin, and J. J. Riera, "Distributions of irritative zones are related to individual alterations of resting-state networks in focal epilepsy," PLos one, 2015.

[37] F. Tadel, S. Baillet, J. C. Mosher, D. Pantazis, and R. M. Leahy, "Brainstorm: a user-friendly application for MEG/EEG analysis," Computational intelligence and neuroscience, vol. 2011, p. 8, 2011.

[38] P. A. Valdés-Hernández, A. Sumiyoshi, H. Nonaka, R. Haga, E. Aubert-Vásquez, T. Ogawa, et al., "An in vivo MRI template set for morphometry, tissue segmentation, and fMRI localization in rats," Frontiers in neuroinformatics, vol. 5, 2011.

[39] G. W. Paxinos, Charles, The rat brain in stereotaxic coordinates, six edition ed.: Academic Press, 2007.

[40] J. Gotman, "Epileptic networks studied with EEG - fMRI," Epilepsia, vol. 49, pp. 42-51, 2008.

[41] T. Ogawa, J. Riera, T. Goto, A. Sumiyoshi, H. Nonaka, K. Jerbi, et al., "Large-scale heterogeneous representation of sound attributes in rat primary auditory cortex: from unit activity to population dynamics," The Journal of Neuroscience, vol. 31, pp. 14639-14653, 2011.

[42] A. K. Dunn, A. Devor, A. M. Dale, and D. A. Boas, "Spatial extent of oxygen metabolism and hemodynamic changes during functional activation of the rat somatosensory cortex," Neuroimage, vol. 27, pp. 279-290, 2005.

[43] M. Kohl, U. Lindauer, G. Royl, M. Kühl, L. Gold, A. Villringer, et al., "Physical model for the spectroscopic analysis of cortical intrinsic optical signals," Physics in medicine and biology, vol. 45, pp. 3749-3764, 2000.

[44] P. Baraldi, A. A. Manginelli, M. Maieron, D. Liberati, and C. A. Porro, "An ARX model-based approach to trial by trial identification of fMRI-BOLD responses," Neuroimage, vol. 37, pp. 189-201, 2007.

[45] R. Jolivet, R. Kobayashi, A. Rauch, R. Naud, S. Shinomoto, and W. Gerstner, "A benchmark test for a quantitative assessment of simple neuron models," Journal of neuroscience methods, vol. 169, pp. 417-424, 2008.

[46] J. Riera, E. Aubert, K. Iwata, R. Kawashima, X. Wan, and T. Ozaki, "Fusing EEG and fMRI based on a bottom-up model: inferring activation and effective connectivity in neural masses," Philosophical Transactions of the Royal Society of London B: Biological Sciences, vol. 360, pp. 1025-1041, 2005.

[47] J. J. Riera, J. C. Jimenez, X. Wan, R. Kawashima, and T. Ozaki, "Nonlinear local electrovascular coupling. II: From data to neuronal masses," Human brain mapping, vol. 28, pp. 335-354, 2007.

[48] L. Tyvaert, C. Hawco, E. Kobayashi, P. LeVan, F. Dubeau, and J. Gotman, "Different structures involved during ictal and interictal epileptic activity in malformations of cortical development: an EEG-fMRI study," Brain, vol. 131, pp. 2042-2060, 2008.

[49] M. Zhao, H. Ma, M. Suh, and T. H. Schwartz, "Spatiotemporal dynamics of perfusion and oximetry during ictal discharges in the rat neocortex," The Journal of Neuroscience, vol. 29, pp. 2814-2823, 2009.

[50] J. K. Thompson, M. R. Peterson, and R. D. Freeman, "Single-neuron activity and tissue oxygenation in the cerebral cortex," Science, vol. 299, pp. 1070-1072, 2003.

[51] H. Choi, Y. K. Kim, H. Kang, H. Lee, H.-J. Im, E. E. Kim, et al., "Abnormal metabolic connectivity in the pilocarpine-induced epilepsy rat model: a multiscale network analysis based on persistent homology," NeuroImage, vol. 99, pp. 226-236, 2014.

[52] B. Jupp, J. Williams, D. Binns, R. J. Hicks, L. Cardamone, N. Jones, et al., "Hypometabolism precedes limbic atrophy and spontaneous recurrent seizures in a rat model of TLE," Epilepsia, vol. 53, pp. 1233-1244, 2012.

[53] G. H. Glover, "Deconvolution of Impulse Response in Event-Related BOLD fMRI 1," Neuroimage, vol. 9, pp. 416-429, 1999.

[54] K. J. Worsley, C. Liao, J. Aston, V. Petre, G. Duncan, F. Morales, et al., "A general statistical analysis for fMRI data," Neuroimage, vol. 15, pp. 1-15, 2002.

[55] F. Foschum and A. Kienle, "Broadband absorption spectroscopy of turbid media using a dual step steady-state method," Journal of biomedical optics, vol. 17, pp. 0370091-0370097, 2012.

[56] K. Hamandi, H. Laufs, U. Nöth, D. W. Carmichael, J. S. Duncan, and L. Lemieux, "BOLD and perfusion changes during epileptic generalised spike wave activity," Neuroimage, vol. 39, pp. 608-618, 2008.

[57] J. Jacobs, E. Kobayashi, R. Boor, H. Muhle, W. Stephan, C. Hawco, et al., "Hemodynamic responses to interictal epileptiform discharges in children with symptomatic epilepsy," Epilepsia, vol. 48, pp. 2068-2078, 2007.

[58] J. Jacobs, P. LeVan, F. Moeller, R. Boor, U. Stephani, J. Gotman, et al.,
"Hemodynamic changes preceding the interictal EEG spike in patients with focal epilepsy investigated using simultaneous EEG-fMRI," Neuroimage, vol. 45, pp. 1220-1231, 2009.

[59] R. C. Thornton, R. Rodionov, H. Laufs, S. Vulliemoz, A. Vaudano, D. Carmichael, et al., "Imaging haemodynamic changes related to seizures: comparison of EEG-based general linear model, independent component analysis of fMRI and intracranial EEG," Neuroimage, vol. 53, pp. 196-205, 2010 .

[60] S. Storti, E. Formaggio, A. Bertoldo, P. Manganotti, A. Fiaschi, and G. Toffolo, "Modelling hemodynamic response function in epilepsy," Clinical Neurophysiology, vol. 124, pp. 2108-2118, 2013. 